\title{
AT1R blocker losartan attenuates intestinal epithelial cell apoptosis in a mouse model of Crohn's disease
}

\author{
TIAN-JING LIU ${ }^{1,2 *}$, YONG-YAN SHI ${ }^{3 *}$, EN-BO WANG ${ }^{1}$, TONG ZHU ${ }^{1,2}$ and QUN ZHAO ${ }^{1,2}$ \\ ${ }^{1}$ Department of Pediatric Orthopedics; ${ }^{2}$ Key Laboratory of Health Ministry for Congenital Malformation; \\ ${ }^{3}$ Department of Pediatrics, Shengjing Hospital of China Medical University, Shenyang, Liaoning 110004, P.R. China
}

Received December 8, 2014; Accepted October 2, 2015

DOI: $10.3892 / \mathrm{mmr} .2015 .4686$

\begin{abstract}
Angiotensin II, which is the main effector of the renin-angiotensin system, has an important role in intestinal inflammation via the angiotensin II type 1 receptor (AT1R). The present study aimed to investigate the protective effects of the AT1R blocker losartan on 2,4,6-trinitrobenzenesulphonic acid (TNBS)-induced colitis. Losartan was administered to male adult C57BL/6 J mice 2 weeks prior to the induction of colitis, and images of the whole colon were captured to record changes, scored according to a microscopic scoring system, and reverse transcription-quantitative polymerase chain reaction were performed in order to investigate colonic inflammation. In addition, intestinal epithelial barrier permeability was evaluated, and intestinal epithelial cell (IEC) apoptosis was measured using terminal deoxynucleotidyl transferase dUTP nick end labeling (TUNEL) staining, and apoptosis-related protein expression levels were detected by western blotting. Losartan was able to attenuate TNBS-induced body weight loss and colonic damage. Furthermore, T helper 1-mediated proinflammatory cytokines were suppressed by losartan, and gut permeability was largely preserved. TUNEL staining revealed reduced IEC apoptosis in the losartan-treated mice. Losartan also increased the B-cell lymphoma 2 (Bcl-2)/Bcl-2-associated $\mathrm{X}$ protein (Bax) ratio and suppressed caspase-3 induction. These results suggested that the AT1R blocker losartan may attenuate TNBS-induced colitis by inhibiting the apoptosis of IECs. The effects of losartan were partially mediated through increasing the $\mathrm{Bcl}-2 / \mathrm{Bax}$ ratio and subsequently suppressing the induction of the proapoptotic mediator caspase- 3 .
\end{abstract}

Correspondence to: Professor Qun Zhao, Department of Pediatric Orthopedics, Shengjing Hospital of China Medical University, 36 Sanhao Street, Shenyang, Liaoning 110004, P.R. China

E-mail: zhaoqunorthopedics@hotmail.com

${ }^{*}$ Contributed equally

Key words: angiotensin II type 1 receptor blocker, Crohn's disease, apoptosis, permeability

\section{Introduction}

Ulcerative colitis and Crohn's disease (CD) are two major types of inflammatory bowel disease (IBD), which influence millions of individuals $(1,2)$; however, the pathogenesis and etiology of IBD remain to be elucidated. Pathological alterations associated with IBD include gut inflammation, disruption of the intestinal epithelial barrier and ulcer formation $(1,2)$. The intestinal epithelial barrier consists of a monolayer of epithelial cells with intercellular tight junctions, which regulates gut permeability (3). Excessive apoptosis of intestinal epithelial cells (IECs) may compromise the intestinal barrier function, and has been recognized as a major pathogenic mechanism in the process of chronic intestinal inflammation (3).

Angiotensin II is a proinflammatory peptide that is associated with digestive system disorders, particularly inflammation (4-7). Previous studies have demonstrated that colonic mucosal levels of angiotensin I and II are significantly elevated in patients with $\mathrm{CD}(4,5)$. In addition, amelioration of 2,4,6-trinitrobenzenesulphonic acid (TNBS)-induced colitis was observed in angiotensinogen knockout mice (8), and dextran sulfate sodium (DSS)-induced colonic inflammation was reduced in angiotensin II type 1 receptor (AT1R)-deficient mice (9). However, little is currently known regarding the possible pathological mechanism of the angiotensin II-AT1R pathway in intestinal inflammation.

Angiotensin II has previously been demonstrated to induce apoptosis via AT1R in myocytes and IECs, and the underlying mechanism may be partially due to its regulatory effect on the B-cell lymphoma 2 (Bcl-2)/Bcl-2-associated X protein (Bax) pathway $(10,11)$. Bax and Bcl-2 are important proapoptotic and anti-apoptotic mediators, respectively, which have a crucial role in regulating IEC apoptosis via the intrinsic apoptotic pathway (12-14). The ratio of Bcl-2 to Bax is frequently used as an indicator of survival potential, in which a high ratio protects against apoptosis and a low ratio promotes apoptosis (12-14).

Although the AT1R blocker losartan has been reported to inhibit the apoptosis of IECs in vitro (11), its effects in vivo, as well as the underlying mechanism, remain to be clarified. The present study investigated the influence of losartan treatment on TNBS-induced colitis, which is a common mouse model of CD (15). It was hypothesized that losartan could attenuate TNBS-induced colitis by inhibiting the apoptosis of IECs, through upregulating the ratio of $\mathrm{Bcl}-2$ to Bax. 


\section{Materials and methods}

Mouse model of TNBS-induced colitis. Male adult C57BL/6 J mice, (8-12 weeks old; 20-25 g), were purchased from the Center for Experimental Animals of China Medical University (Shenyang, China). The animals were housed in specific-pathogen free conditions. Mice were provided with food and water ad libitum and maintained in a $12 \mathrm{~h}$ dark/light cycle at $25^{\circ} \mathrm{C}$. All animal procedures were reviewed and approved by the Institutional Ethical Committee of China Medical University. Mice were anesthetized by an intraperitoneal injection with a cocktail of xylazine (Rompun 2\%; Bayer AG, Leverkusen, Germany) and ketamine (Ketavest; $100 \mathrm{mg} / \mathrm{ml}$; Pfizer, Inc., New York, NY, USA). TNBS was prepared by dissolving 5\% TNBS (Sigma-Aldrich, St. Louis, MO, USA) in an equal volume of $100 \%$ ethanol, in order to generate a working solution of $2.5 \%$ TNBS in $50 \%$ ethanol. To induce colitis, the mice were administered $100 \mathrm{mg} / \mathrm{kg}$ TNBS into the rectum using an 18-gauge stainless steel gavage needle. The vehicle group was given the same volume of $50 \%$ ethanol.

Losartan treatment. Losartan (Cozaar, Merck \& Co., Whitehouse Station, NJ, USA) was administered orally in distilled drinking water ( $\sim 10 \mathrm{mg} / \mathrm{kg} /$ day $)$ for 2 weeks prior to the induction of colitis, and after TNBS administration until the time of sacrifice. This dose was similar to that used in previous studies regarding this drug (16). The vehicle group was given distilled drinking water only.

Western blotting. The mice were sacrificed by cervical dislocation following anesthetization with carbon dioxide, on day 2 after TNBS treatment. The colon was cut open and washed with phosphate-buffered saline, following which the colonic mucosa was harvested and homogenized in radioimmunoprecipitation assay buffer (Beyotime Institute of Biotechnology, Haimen, China). The supernatant was used for the measurement of protein concentration using a bicinchoninic acid assay (Beyotime Institute of Biotechnology). Subsequently, 3X SDS was added and the mixture was heated to $95^{\circ} \mathrm{C}$ for $5 \mathrm{~min}$. The protein lysates $(50 \mu \mathrm{g} / \mathrm{lane})$ were separated by $10 \%$ sodium dodecyl sulfate-polyacrylamide gel electrophoresis, and the proteins were electrophoretically transferred onto polyvinylidene difluoride membranes (EMD Millipore, Billerica, MA, USA). The membranes were blocked with $5 \%$ non-fat milk at room temperature for $1 \mathrm{~h}$, and then washed three times in $0.1 \%$ Tris-buffered saline with Tween 30, with agitation for $10 \mathrm{~min}$. The membranes were then incubated with primary and secondary antibodies. The blots were visualized using an enhanced chemiluminescence kit (Santa Cruz Biotechnology, Inc.) and Image J software (1.47v; National Institutes of Health, Bethesda, MD, USA) was used to measure the density of the bands, which were normalized to $\beta$-actin. The antibodies used in the present study included: Mouse monoclonal anti- $\beta$-actin (cat. no. A1978; 1:10,000; Sigma-Aldrich), rabbit polyclonal anti-Bcl-2 (cat. no. 2876, Cell Signaling Technology, Inc., Beverly, MA, USA; 1:2,000), rabbit polyclonal anti-Bax (cat. no. 2772; 1:2,000; Cell Signaling Technology, Inc.) and rabbit polyclonal anti-caspase 3 (cat. no. 9662; 1:1,000; Cell Signaling Technology, Inc.).
Reverse transcription-quantitative polymerase chain reaction (RT-qPCR). Mice were sacrificed on day 2 after TNBS treatment. A section of distal colon, $\sim 1.0 \mathrm{~cm}$ in length, was harvested from the same segment in all of the mice and RNA was isolated from the colonic mucosa using TRIzol ${ }^{\circledR}$ (Invitrogen; Thermo Fisher Scientific, Inc., Waltham, MA, USA). First-strand cDNA was synthesized from $2 \mu \mathrm{g}$ total RNA in a $20 \mu 1$ reaction system using M-MLV reverse transcriptase (Thermo Fisher Scientific, Inc.) and random primers (Takara Bio, Inc., Otsu, Japan). qPCR was performed using a Roche LightCycler Real Time PCR system with SYBR green PCR Master mix (Takara Bio, Inc., Otsu, Japan). Relative mRNA transcription levels were calculated according to the $2^{-\triangle \Delta C q}$ formula. $\beta-2$ microglobulin was used as an internal control. PCR primer sequences are presented in Table I. The PCR reaction conditions were as follows: $95^{\circ} \mathrm{C}$ for $2 \mathrm{~min}$, followed by 40 cycles of $95^{\circ} \mathrm{C}$ for $15 \mathrm{sec}, 55^{\circ} \mathrm{C}$ for $15 \mathrm{sec}$, $72^{\circ} \mathrm{C}$ for $1 \mathrm{~min}, 72^{\circ} \mathrm{C}$ for $5 \mathrm{~min}$ and then kept at $4^{\circ} \mathrm{C}$ until tubes were removed.

Hematoxylin \& eosin staining. The mice were sacrificed 4 days after TNBS treatment, and images of the colonic morphology were captured using a Sony digital camera Sony digital camera (DSC-TX9C; Sony Corporation, Tokyo, Japan). The damage was scored according to a macroscopic scoring system (17). The distal colon was harvested and fixed overnight with $4 \%$ formaldehyde, dehydrated with graded alcohol, placed in xylene and embedded in paraffin. Sections $(4 \mu \mathrm{m})$ were stained with hematoxylin \& eosin (Beyotime Institute of Biotechnology). Five areas were randomly selected in each section and examined at X100 magnification. In each field, colon microscopic scoring (Leica DFC425; Leica Microsystems GmbH, Wetzlar, Germany) was performed by two pathologists independently that were blind to the study design, according to the following microscopic scoring system (17): Loss of mucosal architecture, cellular infiltration and muscle thickening were scored $0,1,2$ or 3 (absent, mild-severe); crypt abscess formation and goblet cell depletion were scored 0 or 1 (absent or present).

Terminal deoxynucleotidyl transferase dUTP nick end labeling (TUNEL) staining. Apoptotic cells in the colonic sections were identified according to the TUNEL staining method using a commercial kit (Roche Diagnostics Corp., Indianapolis, IN, USA), according to the manufacturer's protocol. Apoptotic index was defined as the percentage of TUNEL-positive-cells (red staining) in 100 randomly chosen IECs, as previously described (18).

Measurement of intestinal permeability. Mice were denied access to food, but were allowed water for $4 \mathrm{~h}$ prior to gavage. At $45 \mathrm{~h}$ post-TNBS injection, $50 \mathrm{mg} / \mathrm{ml}$ fluorescein isothiocyanate-4 kD dextran (FD4; Sigma-Aldrich) was gavaged at $4 \mu \mathrm{l} / \mathrm{g}$ body weight through an 18 -guage stainless steel gavage needle. After $3 \mathrm{~h}$ ( $48 \mathrm{~h}$ post-TNBS injection), the mice were anesthetized via intraperitoneal injection with a cocktail of xylazine and ketamine. Blood was collected from the inferior vena cava with a $1 \mathrm{ml}$ syringe and rested overnight in $4^{\circ} \mathrm{C}$. The blood was then centrifuged at $10,000 \times \mathrm{g}$ for $10 \mathrm{~min}$ at $4^{\circ} \mathrm{C}$, the supernatant was collected and blood serum was harvested, of which $200 \mu 1$ was added to each 
Table I. Primer sequences used for reverse transcription-quantitative polymerase chain reaction.

\begin{tabular}{lll}
\hline Primer name & \multicolumn{1}{c}{ Forward $\left(5^{\prime}-3^{\prime}\right)$} & Reverse $\left(3^{\prime}-5^{\prime}\right)$ \\
\hline TNF- $\alpha$ & ATGAGCACAGAAAGCATGA & AGTAGACAGAAGAGCGTGGT \\
IFN- $\gamma$ & TTCTTCAGCAACAGCAAGGC & TCAGCAGCGACTCCTTTTCC \\
IL-1 $\beta$ & AATGAAAGACGGCACACCCA & TGCTTGTGAGGTGCTGATGT \\
IL-2 & TTGTGCTCCTTGTCAACAGC & CTGGGGAGTTTCAGGTTCCT \\
IL-6 & CCTCTGGTCTTCTGGAGTACC & ACTCCTTCTGTGACTCCAGC \\
IL-12 & GCACCAAATTACTCCGGACG & TGGTCCAGTGTGACCTTCTC \\
IL-17 & TCTCCACCGCAATGAAGACC \\
$\beta-2$ microglobulin & GCTGTGCCTAGGAGTAGCAG & CACACCCACCAGCATCTTCT \\
& CGGCCTGTATGCTATCCAGA & TGGCTGTTGTCCTTGAGTCC
\end{tabular}

TNF, tumor necrosis factor; IFN, interferon; IL, interleukin.

A

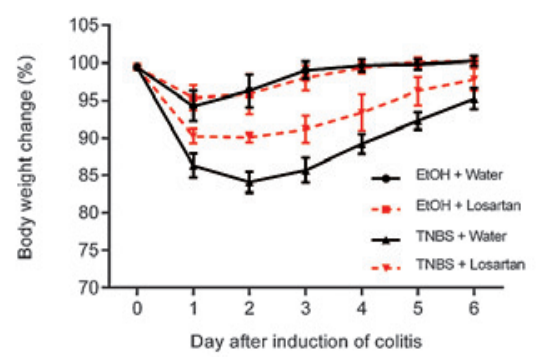

$\mathbf{C}$

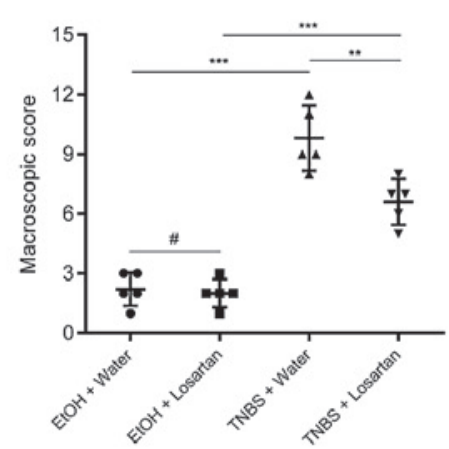

B

EtOH

EtOH TNBS

Water

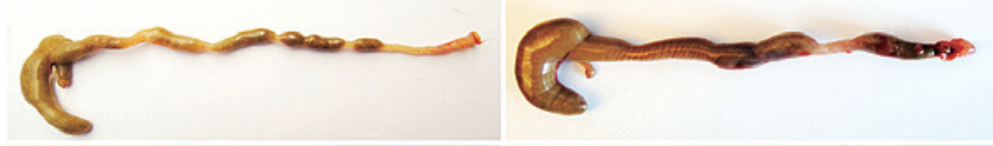

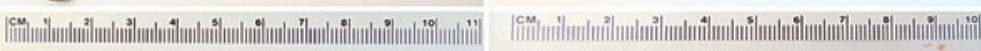

Losartan
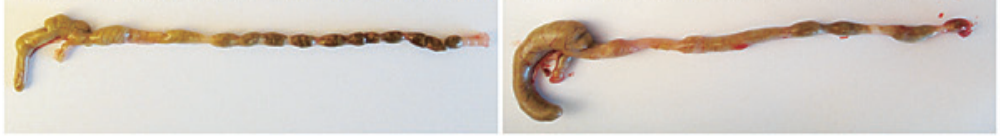

D

$\mathrm{EtOH}$

TNBS

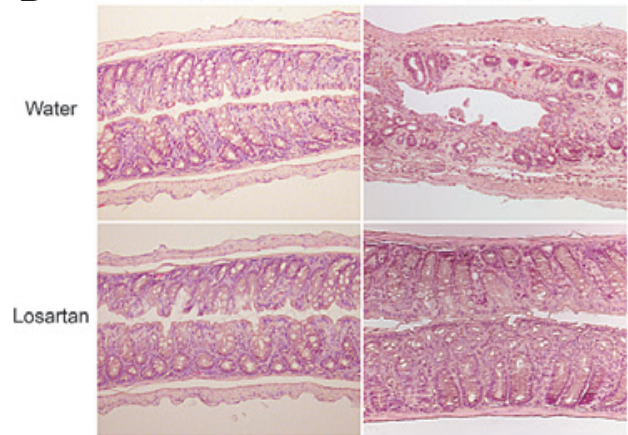

E

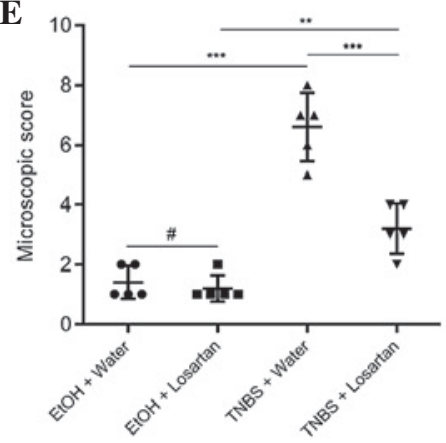

Figure 1. Losartan alleviated 2,4,6-trinitrobenzenesulphonic acid (TNBS)-induced colitis. (A) Body weight change (percentage of original body weight) over time following various treatment methods ( $\mathrm{n}=5$ /group). ${ }^{*} \mathrm{P}<0.05,{ }^{* *} \mathrm{P}<0.01$ and ${ }^{* * *} \mathrm{P}<0.001$ vs. the TNBS + Water group. (B) Representative colon morphology on day 4 after TNBS or ethanol (EtOH) injection. (C) Macroscopic score according to colonic morphology. (D) Representative hematoxylin \& eosin staining. Original magnification, $x 100$. (E) Microscopic scoring of each colon ( $\mathrm{n}=5 /$ group). ${ }^{\#} \mathrm{P}>0.05,{ }^{* *} \mathrm{P}<0.01$ and ${ }^{* * *} \mathrm{P}<0.001$.

well of a 96-well plate. Subsequently, the serum concentration of FD4 was measured using a Synergy HT plate reader (BioTek Laboratories, Inc., Winooski, VT, USA), as previously described (19).

Statistical analysis. All continuous data are presented as the mean \pm standard deviation. Statistical comparisons of continuous variables between groups were conducted using Student's t-test or one-way analysis of variance. Graphpad Prism software 6.0 (Graphpad Software Inc., La Jolla, CA, USA) and SPSS 17.0 (SPSS, Inc., Chicago, IL, USA) were used to perform data analysis. $\mathrm{P}<0.05$ was considered to indicate a statistically significant difference.

\section{Results}

Effects of losartan on TNBS-induced colitis. TNBS successfully induced colitis, as evidenced by marked weight loss, hyperemia, inflammation and ulcerative necrosis in the colon tissue of the TNBS-treated mice, as compared with the ethanol-treated control group, which exhibited only negligible pathological alterations. TNBS-induced weight loss was ameliorated and the colonic morphology was partly restored following treatment with losartan (Fig. 1A-C). Microscopically, transmural inflammation characterized by infiltration of inflammatory cells, mucosal and submucosal ulcerations, loss of goblet cells and fibrosis was observed throughout the 
A

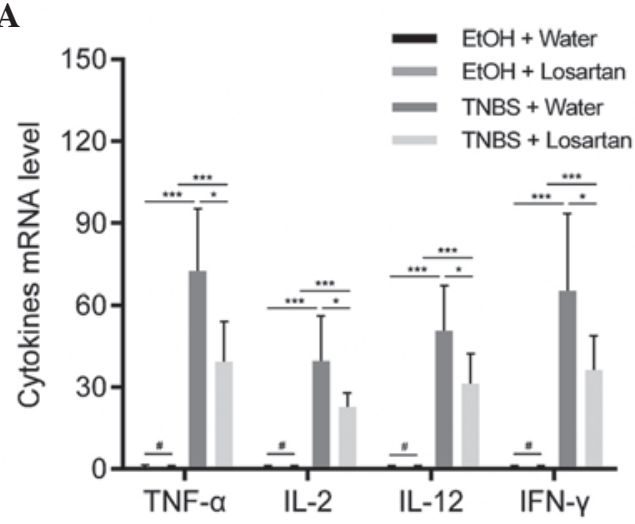

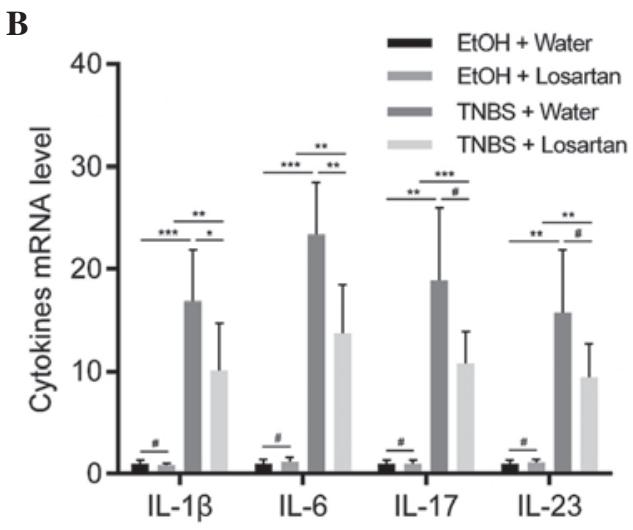

Figure 2. Losartan suppressed 2,4,6-trinitrobenzenesulphonic acid (TNBS)-induced colonic inflammation. Reverse transcription-quantitative polymerase chain reaction was conducted to detect the expression levels of the following proinflammatory cytokines: (A) Tumor necrosis factor (TNF- $\alpha$ ), interleukin (IL)-2, IL-12, interferon (IFN)- $\gamma$, and (B) IL-1 $\beta$, IL-6, IL-17 and IL-23 in the colonic mucosa from each treatment group on day 2 (n=6/group). "P>0.05, ${ }^{*} \mathrm{P}<0.05,{ }^{* *} \mathrm{P}<0.01,{ }^{* * *} \mathrm{P}<0.001 . \mathrm{EtOH}$, ethanol.

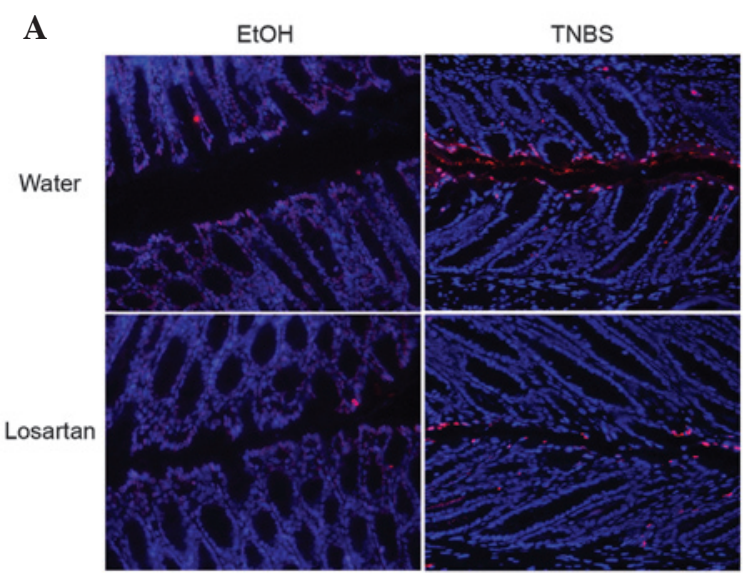

B
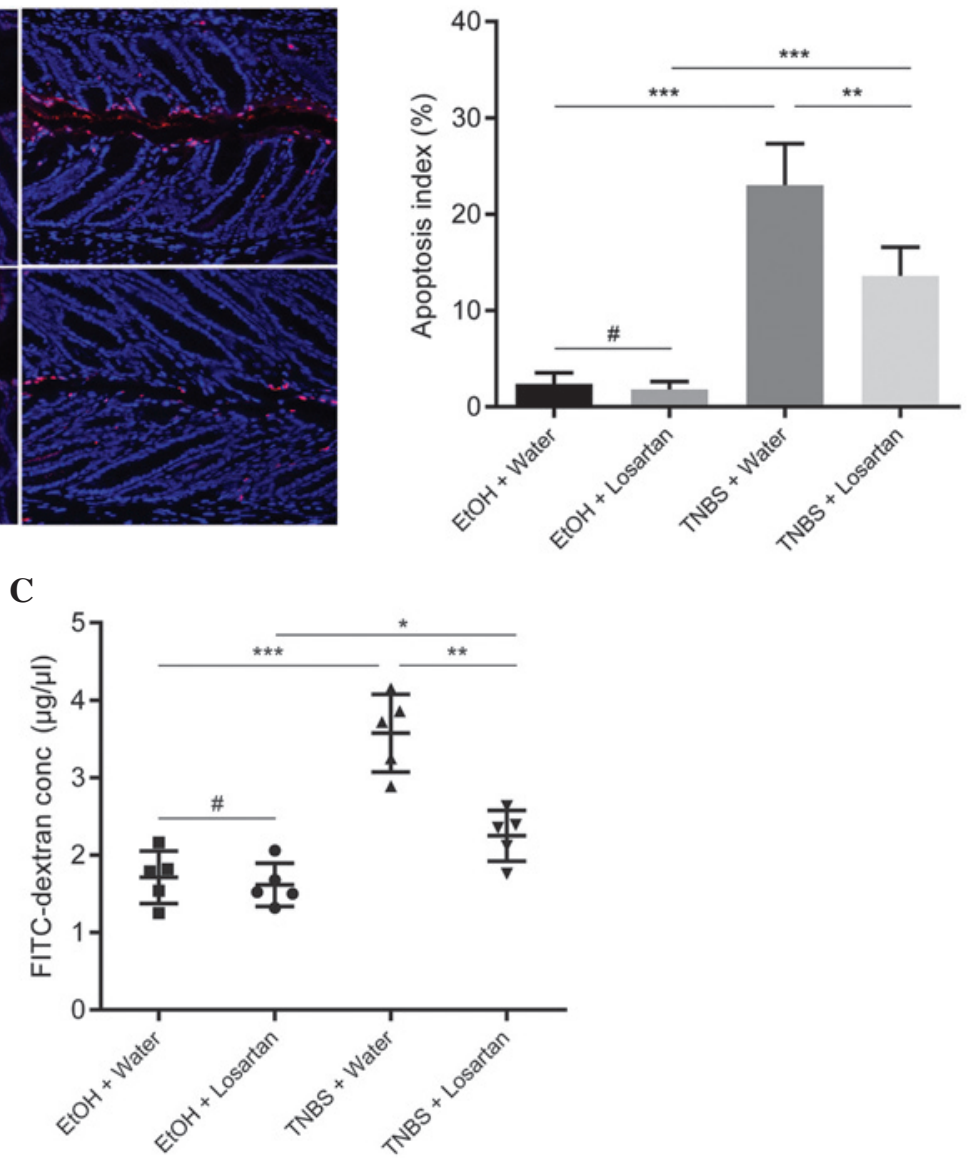

Figure 3. Losartan decreased intestinal permeability by inhibiting intestinal epithelial cell (IEC) apoptosis. (A) Representative terminal deoxynucleotidyl transferase dUTP nick end labeling (TUNEL) staining of each treatment group on day 2 after TNBS treatment. Red spots indicate TUNEL-positive apoptotic IECs. (B) Apoptotic index in each group. Apoptotic index was defined as the percentage of TUNEL-positive cells in 100 randomly selected IECs ( $\mathrm{n}=5 / \mathrm{group}$ ). ${ }^{\#} \mathrm{P}>0.05,{ }^{* *} \mathrm{P}<0.01$ and ${ }^{* * * *} \mathrm{P}<0.001$. (C) Measurement of intestinal permeability ( $\mathrm{n}=5 /$ group). ${ }^{*} \mathrm{P}>0.05,{ }^{*} \mathrm{P}<0.05,{ }^{* *} \mathrm{P}<0.01$ and ${ }^{* * * *} \mathrm{P}<0.001$. TNBS, 2,4,6-trinitrobenzenesulphonic acid; EtOH, ethanol; FITC, fluorescein isothiocyanate.

whole colon in the TNBS-treated mice. Losartan largely rescued the pathological alterations and restored the normal histological structure of the colonic mucosa and submucosa (Fig. 1D and E). These results suggest that losartan may exert protective effects against TNBS-induced colitis.
Losartan suppresses proinflammatory cytokine expression in colonic mucosa. Necrotic inflammation was observed in the colonic mucosa and submucosa of the TNBS-treated mice, which could be partly inhibited by losartan. To further explore the mechanism underlying this effect, the relative expression 
A

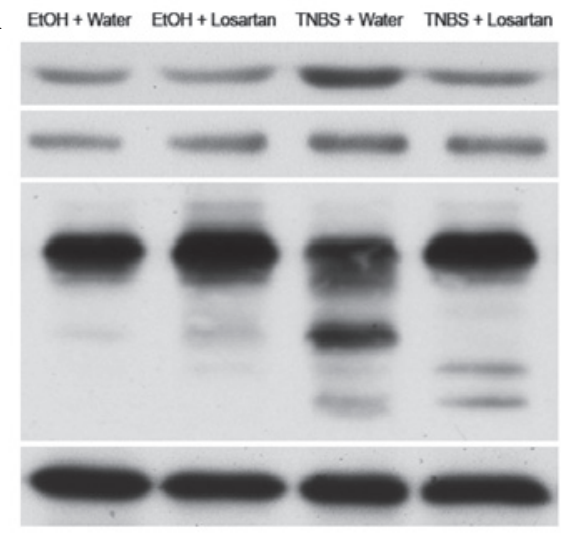

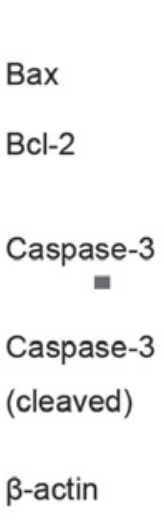

B

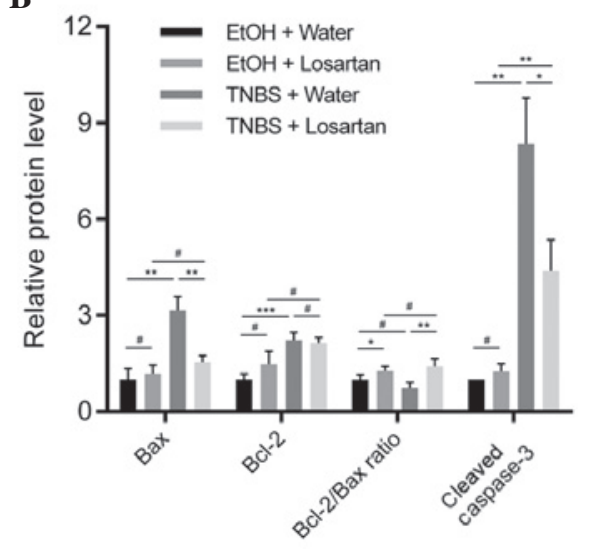

Figure 4. Losartan abrogated caspase-3 induction by increasing the B-cell lymphoma 2 (Bcl-2)/Bcl-2-associated-X protein (Bax) ratio. (A) Western blot analyses and (B) densitometric quantification of anti-apoptotic and proapoptotic proteins in each group ( $\mathrm{n}=4 /$ group). ${ }^{*} \mathrm{P}>0.05,{ }^{*} \mathrm{P}<0.05,{ }^{* *} \mathrm{P}<0.01$ and ${ }^{* * * *} \mathrm{P}<0.001$. TNBS, 2,4,6-trinitrobenzenesulphonic acid; EtOH, ethanol.

levels of proinflammatory cytokines were detected. Since TNBS induces colitis via a Th1-mediated response, production of the following Th1 cytokines was examined: Tumor necrosis factor (TNF)- $\alpha$, interferon (IFN)- $\gamma$, interleukin (IL)-2, IL-12, IL-1 $\beta$ and IL-6 (20). In addition, IL-17 and IL-23, which are derived from Th-17 inflammatory cells, were demonstrated to participate in the inflammatory process of TNBS-induced colitis (20). All of the previously mentioned cytokines were increased following stimulation with TNBS. Losartan was able to significantly ameliorate this tendency for all Th1-mediated cytokines, and was able to decrease the expression levels of the Th17-mediated cytokines but not significantly (Fig. 2).

Losartan decreases intestinal permeability by inhibiting IEC apoptosis. Since losartan had been demonstrated to have an anti-apoptotic effect in vitro (11), the present study investigated whether the protective effect of losartan on TNBS-induced colitis was associated with its anti-apoptotic effect on IECs in vivo. IEC apoptosis may lead to disrupted intestinal barrier function and increased gut permeability, as reflected by increased leakage of FD4 from the lumen into the circulation. An abundance of apoptotic IECs were observed following TNBS administration, whereas the number of apoptotic epithelial cells was markedly reduced in the TNBS + Losartan mice (Fig. 3A and B). Concordantly, treatment with losartan partly alleviated the FD4 leak by decreasing intestinal permeability (Fig. 3C). These results indicate that losartan may preserve the intestinal epithelial barrier and decrease gut permeability by inhibiting the apoptosis of IECs.

Losartan attenuates caspase-3 mediated apoptosis through upregulating the Bcl-2/Bax ratio. The mechanism underlying the anti-apoptotic effect of losartan on IECs was further investigated. Following treatment with TNBS, the expression levels of the proapoptotic protein Bax were markedly increased, whereas the expression levels of the anti-apoptotic protein Bcl-2 were slightly elevated. There was a significant decrease in the protein expression levels of Bax, but not of Bcl-2, in the TNBS + Losartan group, as compared with the TNBS + Water group (Fig. 4A and B), which suggests that losartan treatment may increase the Bcl-2/Bax ratio. Concordantly, the expression levels of cleaved caspase- 3 were markedly inhibited in the TNBS + Losartan group. These data indicate that the anti-apoptotic mechanism of losartan was, at least in part, attributed to the inhibition of caspase- 3 induction through upregulation of the $\mathrm{Bcl}-2 / \mathrm{Bax}$ ratio.

\section{Discussion}

The results of the present study revealed the protective role of the AT1R blocker losartan in TNBS-induced colitis. Losartan was able to relieve the symptoms of TNBS-induced colitis and ameliorate the induction of proinflammatory cytokine expression and IEC apoptosis. Its inhibitory effects on IEC apoptosis were partially mediated through increasing the $\mathrm{Bcl}-2 / \mathrm{Bax}$ ratio and subsequently inhibiting the induction of caspase-3. These observations provided a novel insight into the pathogenesis of TNBS-induced colitis, which serves as a model of CD (15). These results suggest that AT1R blockers may be a potential therapeutic option for patients with IBD.

The classical renin-angiotensin system (RAS) maintains body fluid and electrolyte homeostasis, and a previous study disclosed the link between RAS and IBD (21). Angiotensin II, which is the major biologically active component of the RAS, is involved in apoptosis, vascular remodeling and inflammation (21). Jaszewski et al (4) reported that levels of angiotensin I and II were higher in patients with $\mathrm{CD}$, and this was correlated with the degree of inflammation. Therefore the present study inhibited this pathway using the AT1R blocker losartan, in order to determine whether colonic inflammation could be relieved. Mice treated with losartan exhibited a less severe inflammatory response, as compared with those treated with water only after the administration of TNBS. In addition, the elevation of proinflammatory cytokines was reduced following treatment with losartan.

RAS has an important role in intestinal inflammation in vitro and in vivo. A previous study demonstrated that losartan was able to significantly attenuate angiotensin II-induced IEC apoptosis in cultured HT-29 cell lines (11). Angiotensin converting enzyme inhibitors have been demonstrated to 
markedly attenuate colon inflammation in animal models of colitis $(21,22)$. Furthermore, angiotensinogen gene knockout mice exhibited reduced colitis, as compared with wild type mice following TNBS treatment (8). AT1R-deficient mice also exhibited mild DSS-induced acute colonic inflammation (9).

Angiotensin II has been shown to promote apoptosis through AT1R in numerous circumstances $(10,11)$, including DSS-induced colitis in mice (23). Therefore, preservation of the intestinal epithelial barrier may be due to the anti-apoptotic effect of losartan via blockade of the Angiotensin II-AT1R signaling pathway. Treatment with AT1R blockers has previously been reported to attenuate bleomycin-induced pulmonary epithelial apoptosis in mice (24). In the present study, mice treated with losartan displayed reduced colonic damage following TNBS administration. In addition, the expression levels of caspase-3, a downstream apoptotic protein, were significantly reduced by losartan. These results indicated that losartan was able to attenuate angiotensin II-mediated apoptosis and thus relieve the symptoms of TNBS-induced colitis.

Apoptosis is regulated by a complex interplay between proapoptotic and anti-apoptotic mediators, including Bax and Bcl-2 (12-14). A previous study suggested that angiotensin II-induced IEC apoptosis was associated with the $\mathrm{Bcl}-2 / \mathrm{Bax}$ intrinsic pathway (11). At present, the Bcl-2 protein family is known to consist of 25 homogenous members, including proapoptotic proteins, such as Bcl-2-associated death promoter, $\mathrm{BH} 3$ interacting-domain death agonist and Bax, as well as anti-apoptotic proteins such as Bcl-2, Bcl-extra large and Bcl-2-like protein 2 (14). Bcl-2 suppresses apoptosis by inhibiting the release of cytochrome $c$ from the mitochondria into the cytoplasm (14). The ratio of $\mathrm{Bcl}-2 / \mathrm{Bax}$ is frequently used as an indicator of survival potential, in which a high ratio protects against apoptosis and a low ratio favors apoptosis (12-14). The present study demonstrated that the Bcl-2/Bax ratio was markedly upregulated by losartan, which coincided with a decrease in caspase-3 induction. Regulation of the $\mathrm{Bcl}-2 / \mathrm{Bax}$ intrinsic pathway may contribute to the mechanism underlying the effects of the AT1R pathway on alleviating IEC apoptosis.

Angiotensin II receptors are expressed not only in colonic mucosa (25) but also in microvascular endothelial cells (26). The microvascular system also has an important role in colonic inflammation. Changes in microvascular circulation have been associated with colitis in previous animal studies $(26,27)$, however, colonic blood flow has been reported to be reduced in several colitis models $(27,28,29)$. In the present study, the curative effects of losartan may in part be attributed to its benefits on the microcirculation; however, this requires further investigation.

In conclusion, the present study demonstrated that the AT1R blocker losartan was able to inhibit the apoptosis of IECs, and therefore attenuate TNBS-induced colitis. The effects of losartan may have been mediated, at least in part, through increasing the ratio of Bcl-2/Bax and subsequently suppressing the expression of the proapoptotic mediator caspase-3. Given the active RAS status observed in patients with IBD, AT1R blocker may be a potential therapeutic agent for the treatment of IBD.

\section{Acknowledgements}

The present study was supported by the National Natural Science Foundation of China (grant no. 81271938), Liaoning Science and Technology Project (grant no. 2013225079) and the Outstanding Scientific Fund of Shengjing Hospital.

\section{References}

1. Abraham $\mathrm{C}$ and Cho JH: Inflammatory bowel disease. N Engl J Med 361: 2066-2078, 2009.

2. Podolsky DK: Inflammatory bowel disease. N Engl J Med 347: 417-429, 2002

3. Peterson LW and Artis D: Intestinal epithelial cells: Regulators of barrier function and immune homeostasis. Nat Rev Immunol 14: 141-153, 2014.

4. Jaszewski R, Tolia V, Ehrinpreis MN, Bodzin JH, Peleman RR, Korlipara R and Weinstock JV: Increased colonic mucosal angiotensin I and II concentrations in Crohn's colitis. Gastroenterology 98: 1543-1548, 1990.

5. Garg M, Burrell LM, Velkoska E, Griggs K, Angus PW, Gibson PR and Lubel JS: Upregulation of circulating components of the alternative renin-angiotensin system in inflammatory bowel disease: A pilot study. J Renin Angiotensin Aldosterone Syst 16: 559-569, 2015.

6. Bregonzio C, Armando I, Ando H, Jezova M, Baiardi G and Saavedra JM: Anti-inflammatory effects of angiotensin II AT1 receptor antagonism prevent stress-induced gastric injury. Am J Physiol Gastrointest Liver Physiol 285: G414-G423, 2003.

7. Kuno A, Yamada T, Masuda K, Ogawa K, Sogawa M, Nakamura S, Nakazawa T, Ohara $\mathrm{H}$, Nomura T, Joh T, et al: Angiotensin-converting enzyme inhibitor attenuates pancreatic inflammation and fibrosis in male Wistar Bonn/Kobori rats. Gastroenterology 124: 1010-1019, 2003.

8. Inokuchi Y, Morohashi T, Kawana I, Nagashima Y, Kihara M and Umemura S: Amelioration of 2,4,6-trinitrobenzene sulphonic acid induced colitis in angiotensinogen gene knockout mice. Gut 54: 349-356, 2005.

9. Katada K, Yoshida N, Suzuki T, Okuda T, Mizushima K, Takagi T, Ichikawa H, Naito Y, Cepinskas G and Yoshikawa T: Dextran sulfate sodium-induced acute colonic inflammation in angiotensin II type 1a receptor deficient mice. Inflamm Res 57: 84-91, 2008.

10. Leri A, Claudio PP, Li Q, Wang X, Reiss K, Wang S, Malhotra A, Kajstura J and Anversa P: Stretch-mediated release of angiotensin II induces myocyte apoptosis by activating p53 that enhances the local renin-angiotensin system and decreases the Bcl-2-to-Bax protein ratio in the cell. J Clin Invest 101: 1326-1342, 1998.

11. Wang W, Sun L, Xiao W and Yang H: Essential role of angiotensin receptors in the modulation of intestinal epithelial cell apoptosis. J Pediatr Gastroenterol Nutr 57: 562-569, 2013.

12. Pawlowski J and Kraft AS: Bax-induced apoptotic cell death. Proc Natl Acad Sci USA 97: 529-531, 2000.

13. Tang Y, Swartz-Basile DA, Swietlicki EA, Yi L, Rubin DC and Levin MS: Bax is required for resection-induced changes in apoptosis, proliferation, and members of the extrinsic cell death pathways. Gastroenterology 126: 220-230, 2004.

14. Czabotar PE, Lessene G, Strasser A and Adams JM: Control of apoptosis by the BCL-2 protein family: Implications for physiology and therapy. Nat Rev Mol Cell Biol 15: 49-63, 2014.

15. Morris GP, Beck PL, Herridge MS, Depew WT, Szewczuk MR and Wallace JL: Hapten-induced model of chronic inflammation and ulceration in the rat colon. Gastroenterology 96: 795-803, 1989.

16. Wengrower D, Zanninelli G, Latella G, Necozione S, Metanes I, Israeli E, Lysy J, Pines M, Papo O and Goldin E: Losartan reduces trinitrobenzene sulphonic acid-induced colorectal fibrosis in rats. Can J Gastroenterol 26: 33-39, 2012.

17. Appleyard CB and Wallace JL: Reactivation of hapten-induced colitis and its prevention by anti-inflammatory drugs. Am J Physiol 269: G119-G125, 1995.

18. Liu W, Chen Y, Golan MA, Annunziata ML, Du J, Dougherty U, Kong J, Musch M, Huang Y, Pekow J, et al: Intestinal epithelial vitamin D receptor signaling inhibits experimental colitis. J Clin Invest 123: 3983-3996, 2013. 
19. Su L, Nalle SC, Shen L, Turner ES, Singh G, Breskin LA, Khramtsova EA, Khramtsova G, Tsai PY, Fu YX, et al: TNFR2 activates MLCK-dependent tight junction dysregulation to cause apoptosis-mediated barrier loss and experimental colitis. Gastroenterology 145: 407-415, 2013.

20. Brand S: Crohn's disease: Th1, Th17 or both? The change of a paradigm: New immunological and genetic insights implicate Th17 cells in the pathogenesis of Crohn's disease. Gut 58: 1152-1167, 2009.

21. Hume GE and Radford-Smith GL: ACE inhibitors and angiotensin II receptor antagonists in Crohn's disease management. Expert Rev Gastroenterol Hepatol 2: 645-651, 2008.

22. Spencer AU, Yang H, Haxhija EQ, Wildhaber BE, Greenson JK and Teitelbaum DH: Reduced severity of a mouse colitis model with angiotensin converting enzyme inhibition. Dig Dis Sci 52: 1060-1070, 2007.

23. Okawada M, Koga H, Larsen SD, Showalter HD, Turbiak AJ, Jin X, Lucas PC, Lipka E, Hillfinger J, Kim JS and Teitelbaum DH: Use of enterally delivered angiotensin II type 1a receptor antagonists to reduce the severity of colitis. Dig Dis Sci 56: 2553-2565, 2011.

24. Li X, Rayford H and Uhal BD: Essential roles for angiotensin receptor AT1a in bleomycin-induced apoptosis and lung fibrosis in mice. Am J Pathol 163: 2523-2530, 2003.
25. Hirasawa K, Sato Y, Hosoda Y, Yamamoto T and Hanai H: Immunohistochemical localization of angiotensin II receptor and local renin-angiotensin system in human colonic mucosa. J Histochem Cytochem 50: 275-282, 2002.

26. Petnehazy T, Cooper D, Stokes KY, Russell J, Wood KC and Granger DN: Angiotensin II type 1 receptors and the intestinal microvascular dysfunction induced by ischemia and reperfusion. Am J Physiol Gastrointest Liver Physiol 290: G1203-G1210, 2006.

27. Mori M, Stokes KY, Vowinkel T, Watanabe N, Elrod JW, Harris NR, Lefer DJ, Hibi T and Granger DN: Colonic blood flow responses in experimental colitis: Time course and underlying mechanisms. Am J Physiol Gastrointest Liver Physiol 289: G1024-G1029, 2005.

28. Deniz M, Cetinel S and Kurtel H: Blood flow alterations in TNBS-induced colitis: Role of endothelin receptors. Inflamm Res 53: 329-336, 2004.

29. Garrelds IM, Heiligers JP, Van Meeteren ME, Duncker DJ, Saxena PR, Meijssen MA and Zijlstra FJ: Intestinal blood flow in murine colitis induced with dextran sulfate sodium. Dig Dis Sci 47: 2231-2236, 2002. 